Cahiers $d u$ MONDE RUSSE

\section{Cahiers du monde russe}

Russie - Empire russe - Union soviétique et États indépendants

$51 / 4 \mid 2010$

Sciences humaines et sociales en Russie à l'Âge d'argent

\title{
Melissa L. Caldwell, Dacha Idylls
}

\section{Ronan Hervouet}

\section{(2) OpenEdition}

1 Journals

\section{Édition électronique}

URL : https://journals.openedition.org/monderusse/7402

DOI : 10.4000/monderusse. 7402

ISSN : $1777-5388$

Éditeur

Éditions de l'EHESS

\section{Édition imprimée}

Date de publication : 25 novembre 2010

Pagination : 740-743

ISBN : 978-2-7132-2316-7

ISSN : $1252-6576$

Référence électronique

Ronan Hervouet, "Melissa L. Caldwell, Dacha Idylls », Cahiers du monde russe [En ligne], 51/4 | 2010, mis en ligne le 09 décembre 2011, consulté le 03 septembre 2022. URL : http://

journals.openedition.org/monderusse/7402; DOI : https://doi.org/10.4000/monderusse.7402

Ce document a été généré automatiquement le 3 septembre 2022

Tous droits réservés 


\title{
Melissa L. Caldwell, Dacha Idylls
}

\author{
Ronan Hervouet
}

\section{RÉFÉRENCE}

Melissa L. CALDWELL, Dacha Idylls. Living Organically in Russia's Countryside.

Berkeley - Los Angeles : University of California Press, 2011, 200 p.

1 Anthropologue à l'université de Californie (Santa Cruz), Melissa Caldwell mène des enquêtes de terrain en Russie depuis le milieu des années 1990 et consacre son dernier ouvrage à « la vie à la datcha ». Retraçant brièvement l'histoire des datchas en Russie depuis l'époque de Pierre de Grand, l'auteur précise qu'elle ne s'intéresse pas aux villégiatures des élites mais aux constructions situées sur des lopins de terre distribués aux citadins par les autorités soviétiques après 1945. Leur fréquentation reste, aujourd'hui encore, un phénomène massif en Russie. L'anthropologue considère que les datchas, loin d'être des espaces anecdotiques situés sur les marges du social, sont au cœur des expériences vécues des Russes. L'analyse des pratiques à l'œuvre dans ces microcosmes, des discours et représentations qui circulent à propos de cette «institution sociale» lui permet d'aborder des questions plus générales comme la transition vers le capitalisme et l'identité nationale en Russie.

Les propos de Melissa Caldwell reposent sur de riches matériaux empiriques. Entre 1995 et 2005, l'auteur a mené des entretiens informels avec "plusieurs centaines de personnes » à la datcha, en ville, et même auprès de Russes émigrés aux États-Unis. Elle a visité une quinzaine de collectifs de datchas dans les environs de Moscou, de SaintPétersbourg et de Tver'. En 2005, elle a séjourné trois mois près de Tver' dans un de ces collectifs, où elle a mené des entretiens formels. La plupart des personnes interviewées sont diplômées du supérieur (médecins, juristes, ingénieurs, musiciens, etc.) et perçoivent des revenus variés - mais l'auteur ne donne aucune précision chiffrée. Leurs datchas ont des statuts juridiques différents: certains sont propriétaires, d'autres continuent de louer leur parcelle à l'État. Melissa Caldwell nous dit qu'ils prétendent partager une «sensibilité de classe moyenne» (p.19), mais elle n'explicite pas cette 
catégorie problématique en sociologie. On découvre à la lecture que les matériaux utilisés sont également composés d'œuvres littéraires, de slogans publicitaires, d'émissions de télévision, de revues de jardinage et d'articles de presse. Toutefois, ce corpus ne fait l'objet d'aucune présentation systématique.

3 Melissa Caldwell nous propose d'abord une description ethnographique détaillée de la « vie à la datcha ». Puis, dans les chapitres II et III, elle analyse les émotions éprouvées dans ce lieu, l'expérience à la datcha se caractérisant par l'épreuve simultanée du plaisir et de la peine. Ses interlocuteurs expriment à la fois la joie d'être à la datcha et les difficultés à s'y rendre, à maintenir en état sa maison et à cultiver son jardin. C'est un espace où se déploient " repos actif» et "loisir sérieux ». Melissa Caldwell se tourne alors vers Čehov et Gor'kij, mais aussi vers les travaux de l'anthropologue Nancy Ries ${ }^{1}$ pour saisir en quoi la présence simultanée de ces émotions contradictoires caractérise une forme d'"intimité culturelle", une sensibilité particulière revendiquée par les Russes : la peine n'est pas seulement une épreuve que l'on subit, mais que l'on endure aussi pour en retirer une certaine fierté.

4 L'une des raisons du plaisir éprouvé à la datcha provient d'une expérience particulière de la temporalité. Plusieurs interlocuteurs prétendent que le rythme de la vie urbaine est la cause de difficultés respiratoires, nausées, maux de tête, hypertension et insomnies, tandis qu'à la datcha le temps semble ralentir, ils dorment mieux et prennent moins de médicaments. L'auteur décrit à ce propos les difficultés de la vie en ville à l'époque de l'URSS (pénuries chroniques, files d'attente, etc.) ainsi que dans la Russie postsoviétique (emplois du temps surchargés, problèmes de transports, sentiment d'insécurité, etc.).

5 Melissa Caldwell revient ensuite sur l'une des origines de la fatigue éprouvée à la datcha, à savoir la culture du potager. À l'époque soviétique, ces jardins ont permis de surmonter les difficultés liées aux pénuries alimentaires et, dans les années 1990, ils ont joué un rôle important dans les « stratégies de survie » des Russes. Mais aujourd'hui, quelle raison a$\mathrm{t}$-on de cultiver un potager? Adoptant les conclusions du sociologue Simon Clarke ${ }^{2}$, l'auteur juge que cette activité n'est plus rentable - il est dommage à ce propos qu'elle ne discute pas le travail de Caleb Southworth ni ne cite son article intitulé « The Dacha Debate. Household Agriculture and Labor Markets in Post-Socialist Russia " (Rural Sociology, 71 (3), 2006, p. 451-478).

6 Dès lors, pourquoi ne pas abandonner la culture du potager? L'auteur mobilise d'abord l'idée selon laquelle les jardiniers éprouvent du plaisir à travailler pour eux-mêmes et non pour l'État ou pour une entreprise. Puis, dans le chapitre IV, elle analyse l'attachement des Russes aux "produits naturels », cultivés sans produits chimiques ni pesticides industriels. Cette inclination est d'abord liée à la perception qu'ils ont de la sécurité alimentaire. Suspicieux à l'égard des produits issus de la filière agroindustrielle, les interviewés jugent « écologiquement propre » ce qui est cultivé et qui circule dans des réseaux personnels. Cette posture traduit plus généralement un jugement sur les transformations capitalistes à l'œuvre en Russie. Comme la sociologue Jane Zavisca ${ }^{3}$ l'avait déjà souligné, les dačniki dénoncent les forces anonymes du marché qui régissent aujourd'hui les transactions et ils valorisent les cycles d'échanges de cadeaux et de dons qui caractérisent, selon la sociologue Alena Ledeneva ${ }^{4}$, l'époque soviétique.

7 Cet attachement aux produits «bio " révèle aussi une représentation particulière de l'identité nationale. Melissa Caldwell parle du « nationalisme géographique » des Russes, qui définissent leur identité en référence à un environnement naturel spécifique. Être 
Russe, c'est en effet pouvoir compter sur sa terre nourricière qui serait plus " propre " qu'aucune autre. Les fruits et légumes qui y poussent naturellement auraient même des vertus purificatrices. En témoigne d'ailleurs la croyance dans leurs propriétés médicinales. Pour beaucoup, mieux vaut d'ailleurs se soigner en consommant ce que l'on a soi-même cultivé que de suivre les prescriptions des médecins dont on se méfie autant que des circuits économiques capitalistes. Si cette sensibilité fait écho à l'engouement actuel pour les philosophies orientales (feng shui) et "New Age", elle se distingue des courants occidentaux d' "éco-gastronomie " comme le mouvement "Slow Food", et plus généralement de l'écologie politique. En effet, cette valorisation des produits naturels ne s'articule pas à une critique du modèle productiviste dans le monde agricole.

Dans le chapitre V, Melissa Caldwell analyse les discours récurrents sur la peur de la disparition du mode de vie "authentique " à la datcha. Si les dačniki se satisfont de l'introduction de certaines innovations qui améliorent leur quotidien (tondeuses à gazon, téléphones mobiles, eau courante, accès à Internet), ils s'inquiètent d'autres changements: crise des campagnes russes, vente de nombreuses datchas (sans que l'auteur ne donne d'indication précise sur l'ampleur de ces transactions), spéculation foncière, violence dans les villes... Ces peurs rejaillissent dans le monde des datchas sous forme de plaintes visant ceux qui n'appartiennent pas à la communauté traditionnelle des dačniki. On critique les "Moscovites» et leurs comportements (mauvais goût, érection de clôtures, bruits, dédain...), sans que le lecteur comprenne précisément qui sont ces « Nouveaux Russes »; les étrangers, sous les traits du "Caucasien» (p. 110); et parfois les jeunes, mais les conflits intergénérationnels sur les usages du jardin sont à peine esquissés par l'auteur. Le monde d'avant les changements apparait dès lors « idyllique » et les transformations en cours suscitent mécaniquement une profusion de discours nostalgiques.

Melissa Caldwell aborde enfin, dans le chapitre VI, la question de la société civile dans le monde des datchas. Celle-ci avait déjà été soulevée dans la thèse de sociologie de Naomi Galt $^{5}$, que l'auteur cite sans toutefois entrer en discussion avec ses arguments. Comme les Russes y négocient des règles et y développent un sens de l'ordre et de la justice, l'anthropologue considère que les valeurs démocratiques de liberté, d'autonomie et d'esprit associatif et, par conséquent, une "forme de société civile»(p.134), se manifestent dans ces espaces. Pour preuve, elle montre que les datchas sont des lieux de discussions incessantes sur le comportement des nouveaux arrivants, la gestion des ordures, l'achat des terres par des entreprises privées, les vols et le vandalisme, les feux de forêts, autant de dangers dont les dačniki trouvent les causes dans l'avènement de l'individualisme et les méfaits de la démocratie. Mais l'auteur ne précise pas pourquoi elle voit de la démocratie là où l'existence même de ce régime politique semble si souvent contestée. Melissa Caldwell inscrit même cette " démocratie » dans une histoire plus longue, se référant à la datcha comme lieu supposé de combat et de résistance à l'époque soviétique.

10 L'ouvrage, riche, documenté et foisonnant, mais qui pèche parfois par son imprécision, atteint néanmoins l'objectif annoncé en introduction : les datchas y apparaissent comme de véritables observatoires de l'inscription de phénomènes structuraux dans les configurations du quotidien. Dacha Idylls nous plonge ainsi dans les mondes vécus des dačniki, nous faisant partager leurs joies et leurs regrets, leurs plaisirs et leurs angoisses. Melissa Caldwell articule ces descriptions de la «vie à la datcha » à des réflexions générales sur la démocratie, la justice et l'identité nationale, démontrant ainsi toute la 
pertinence de la démarche anthropologique pour comprendre les mutations qui affectent la Russie d'aujourd'hui' ${ }^{6}$.

\section{NOTES}

1. Nancy Ries, Russian Talk: Culture and Conversation during Perestroika, Ithaca - Londres : Cornell University Press, 1997.

2. Simon Clarke, Making Ends Meet in Contemporary Russia: Secondary Employment, Subsidiary Agriculture and Social Networks, Cheltenham : Edward Elgar, 2002.

3. Jane Zavisca, "Contesting Capitalism at the Post-Soviet Dacha: The Meaning of Food Cultivation for Urban Russians », Slavic Review, 62 (4), 2008, p. 786-810.

4. Alena Ledeneva, Russia's Economy of Favours: Blat, Networking and Informal Exchange, Cambridge : Cambridge university Press, 1998.

5. Naomi Galtz, «Space and the Everyday : An Historical Sociology of the Moscow Dacha », thèse de doctorat, université du Michigan, 2000.

6. Sur le thème de la datcha, voir notamment: Stephen Lovell, Summerfolk: A History ot the Datcha, 1710-2000, Ithaca - Londres : Cornell UP, 2003 (voir recension CMR, 44(4), oct.-déc. 2003, p. 819-821) ; Vlada Traven, La datcha en Russie de 1917 à nos jours, P. : Éditions du Sextant, 2005 (CMR, 46(4), oct.-déc. 2005, p. 941-942) ; Ronan Hervouet, Datcha blues: Existences ordinaires et dictature en Biélorussie, P. : Belin, coll. « Europes centrales », 2009. 